\title{
PARTICIPACIÓN, COMUNICACIÓN Y NEGOCIACIÓN EN CONFLICTOS AMBIENTALES: ENERGÍA EÓLICA MARINA EN EL MAR DE TRAFALGAR ${ }^{1}$
}

\author{
Marta I. González y Betty Estévez \\ Departamento de Ciencias, Tecnología y Sociedad \\ Instituto de Filosofia, CSIC
}

\begin{abstract}
Wind energy has experienced a dramatic increase in Spain in the last years. As a result, Spain has become one of the leading countries in wind energy production. However, wind plants location has an ambivalent social perception: while, in general, the public consistentely favours the promotion of renovable energies, the opposition to specific projects is very significant. In this paper, we analyse the social debate surrounding a project of offshore wind energy in Cádiz (Southern Spain), on the basis of semistructured interviews with relevant social actors. Factors like previous experience, trust, scientific expertise, local knowledges, and traditional values have played a role in local opposition. From the analysis of the public conflict and the political answers, we reflect upon the problems and tensions of the interaction among politicians, experts, ecologists and citizens involved in environmental conflicts.
\end{abstract}

KEY WORDS Environmental conflicts, public participation, wind energy.
RESUMEN La energía eólica ha experimentado un crecimiento espectacular en España en los últimos años, habiendo alcanzado los puestos de cabeza entre los paises productores a nivel mundial. En el caso de la implantación de proyectos eólicos nos encontramos con una percepción social ambigua: mientras que la mayor parte de la población está decididamente a favor de la promoción de las energías renovables, la oposición a proyectos concretos de parques eólicos es muy significativa. En este trabajo se presenta el análisis llevado a cabo mediante entrevistas semiestructuradas a actores relevantes en el caso del proyecto de desarrollo eólico marítimo en la provincia de Cádiz. En la oposición social suscitada por este proyecto han intervenido factores como la experiencia previa, la confianza entre las partes implicadas, el asesoramiento científico-técnico movilizado, los conocimientos locales, y el valor asociado a actividades tradicionales. A partir de la polémica pública y de la respuesta de la administración se plantean problemas y tensiones de la interacción entre políticos, expertos, asociaciones ecologistas y ciudadanos afectados en casos de conflictos ambientales.

PALABRAS CLAVE Conflictos ambientales, participación pública, energía eólica.

\section{Ciencia, tecnología y conflictos ambientales alrededor de la energía eólica}

Las intervenciones con efectos sobre el medio ambiente son una importante fuente de conflictos públicos relacionados con la ciencia y la tecnología en todo el mundo. Al mismo tiempo, evaluar las posibles consecuencias ambientales de una intervención en determinado emplazamiento requiere el conocimiento de un buen número de factores de naturaleza local. Por estas dos razones, una gestión participativa de los problemas derivados de los efectos medioambientales de la ciencia y la tecnología minimizaria el conflicto y favoreceria la adecuada valoración de todos los factores relevantes.
Los ejemplos referidos a la interacción y conflictos entre politicos, público y expertos en materia de medio ambiente en España son numerosos: repoblaciones forestales, autovías y autopistas, gestión de espacios naturales, desarrollo de infraestructuras... (López Cerezo et al., 1998; López Cerezo y González García, 2002). Para el tratamiento de estas situaciones conflictivas existe una amplia legislación ambiental, consistente prácticamente en su totalidad en la transposición de directivas comunitarias, a la que también se han incorporado disposiciones referidas a la implicación activa del públi$\mathrm{co}^{2}$. Del mismo modo, se han creado Comisiones Asesoras de Medio Ambiente o Consejos temáticos, tanto a nivel estatal como autonómico, en los que participan representantes de 
organizaciones no gubernamentales de defensa del medio ambiente, además de representantes de la administración, la empresa, sindicatos y expertos universitarios.

Sin embargo, el análisis de algunos casos muestra que la legislación es frecuentemente incumplida, o insatisfactoriamente cumplida, considerándose a menudo más un escoIlo a superar que un procedimiento necesario (López Cerezo y González Garcia, 2002; Todt, 2003a y 2003b); y que, incluso cuando es la presión pública la que desencadena la adopción de estrategias de negociación o participación, estas estrategias fracasan a menudo por desconfianza de los implicados, falta de compromiso por parte de la administración y sensación general de inutilidad de los procesos. Atender, por tanto, a las iniciativas tomadas por los gestores, a la estela de las directivas europeas o por demanda social, estudiando su desarrollo y sus resultados, es necesario para identificar tanto riesgos de fracaso como buenas prácticas.

Un caso vigente en España de polémica medioambiental está relacionado con el auge actual de la energía eólica. En los últimos diez años, la energía eólica ha experimentado un impresionante crecimiento a nivel mundial, especialmente en Europa, encontrándose España entre los paises más destacados en producción. Frente a otros desarrollos tecnológicos en el campo de la energía, cuya implementación cuenta de mano con la oposición del público (como es el caso de la energía nuclear), el crecimiento de la energía eólica es la concreción de una vieja demanda de las organizaciones ecologistas en favor de la promoción de fuentes renovables. Sin embargo, su desarrollo no está exento de conflictos de intereses. Mientras políticos, industria y grandes organizaciones ecologistas se encuentran como aliadas a favor del desarrollo eólico, ciudadanos afectados y pequeñas agrupaciones conservacionistas lamentan la colocación indiscriminada de aeorogeneradores al amparo de unas condiciones económicas muy favorables, y sus efectos sobre la fauna, el paisaje, la riqueza arqueológica, el ruido o actividades económicas tradicionales.

La participación pública en la ubicación de parques eólicos ha consistido básicamente hasta el momento en España en un tipo de participación reactiva: presentación de alegaciones a los proyectos diseñados para diferentes emplazamientos. En este sentido, aunque son de valorar los esfuerzos para legislar la aprobación de proyectos y su evaluación de impacto ambiental, incluyendo la posibilidad de presen- tar alegaciones por parte de asociaciones y particulares involucrados, las leyes no parecen cumplir bien su función, la información no siempre llega a los interesados, y las alegaciones bien fundamentadas no siempre son tenidas en cuenta.

Un paso más allá en la diversificación formal de los actores y en su capacidad de intervención sobre los proyectos eólicos se ha dado en la provincia de Cádiz, a raíz del conflicto social producido por el anuncio del plan para la construcción de parques eólicos marinos en la zona de Trafalgar. La Diputación de Cádiz, consciente del alcance de la polémica, organizó el Foro de la Energía Eólica Marina y el Desarrollo Sostenible, un mecanismo deliberativo en el que participaron políticos y técnicos de la administración junto a representantes de los grupos afectados por el conflicto: empresarios, expertos de la Universidad, ecologistas, alcaldes de las poblaciones afectadas y miembros de las cofradías de pescadores. La importante repercusión pública de la polémica y de la iniciativa de la Diputación cumplió en este caso un papel fundamental en la evolución del proyecto.

En este trabajo se exponen, en una primera parte, las características particulares que hacen especialmente interesante el caso de la energía eólica para analizar la diversificación de actores sociales en la toma de decisiones relacionadas con la ciencia, la tecnología y el medio ambiente. En particular, se destacan aspectos de la percepción pública de la energía eólica y los factores que la condicionan. A continuación se repasa la situación del desarrollo eólico en España y, especificamente, en la provincia de Cádiz, con especial atención a los procesos utilizados para implicar al público afectado en la resolución de propuestas. La discusión se centra, finalmente, en el proyecto de explotación eólica maritima en el Mar de Trafalgar, sobre la base de una serie de entrevistas semiestructuradas realizadas a los actores involucrados en la controversia. El análisis de la controversia y de las medidas adoptadas por la administración para su resolución da pie a algunas consideraciones finales acerca de los procesos de gobernanza ambiental en ámbitos locales.

\section{Energía eólica en Europa y España: implantación e integración}

En el problema del abastecimiento energético se combinan de maneras habitualmente complejas aspectos ambienta- 
les, económicos y políticos. La obtención, uso y gestión de la energía son asuntos públicos de primera magnitud. La forma que ha adoptado el desarrollo en el mundo occidental requiere cantidades ingentes de energia que se ha obtenido mayoritariamente de fuentes no renovables como el petróleo o el carbón. Enfrentados al agotamiento de las reservas energéticas y al cambio climático global, la discusión sobre fuentes alternativas de energía es más que nunca necesaria.

En este sentido, la Unión Europea ha tomado como una de sus prioridades en materia energética la promoción de las energías renovables, tanto en relación con la estrategia de Lisboa de proporcionar alta tecnologia a la Unión Europea, como en relación con el cumplimiento del Protocolo de Kioto. En 1997 se diseñó el Libro Blanco "Energía para el futuro: fuentes de energias renovables", cuyo horizonte se centraba en duplicar el uso de las fuentes energéticas renovables para el año 2010. Siguiendo este mismo marco de acciones y en concordancia con las directrices europeas, el Gobierno español aprobó dos años después el «Plan de Fomento de las Energías Renovables en España", elaborado por el Instituto para la Diversificación y Ahorro de la Energía (IDEA) (Martínez, 1999).

De entre las fuentes de energía renovable en desarrollo en la actualidad, la energía eólica es, según el WorldWatch Institute, la forma de energia de crecimiento más rápido en nuestros días (entre un 15\% y un 75\% anualmente durante los últimos cinco años, siendo la media europea de un $40 \%)$. También la Unión Europea promueve la energía eólica como una apuesta por el desarrollo sostenible, el crecimiento económico y el empleo. Entre los paises europeos, son aquellos que han contado con un sistema de apoyo al precio en los que más se ha desarrollado la industria eólica, de tal modo que Alemania, Dinamarca y España concentran el $82 \%$ de la potencia eólica instalada en la Unión Europea (Delás, 2003). Europa es también pionera en el desarrollo de parques eólicos marinos que podrian, según un informe de 2004 de la consultora Garrad Hassan, proporcionar toda la energía residencial de la región en un plazo de 15 años (Brown, 2004).

La implantación de parques eólicos en España ha adquirido relevancia en los últimos años debido a su elevado potencial en el sector, tanto por lo que respecta al viento como recurso como por lo que respecta a su nivel de desarrollo tecnológico. La energía generada por el viento en España ha ido aumentando paulatina y rápidamente desde finales de los años 80. Según el informe de marzo de 2005 del Global Wind Energy Council, España ocupaba a finales de 2004 el segundo puesto mundial, detrás de Alemania y habiendo superado a Dinamarca y Estados Unidos en los últimos dos años ${ }^{3}$.

El asombroso crecimiento de la energía eólica en España durante la última década muestra los efectos positivos de una política de promoción decidida de las energías renovables, y también ha estimulado la discusión sobre actitudes públicas y efectos ambientales. Por otra parte, el traspaso de competencias en materia de energía (Ley 9/1992) a las Comunidades Autónomas supuso un desarrollo muy desequilibrado de la energía eólica en las diferentes comunidades autónomas. Mientras algunos gobiernos autonómicos apostaron muy tempranamente por esta fuente alternativa de energía, en otros se obstaculizó incluso con moratorias encubiertas. En la actualidad, el mayor desarrollo eólico corresponde a las comunidades de Galicia, Castilla-León, Castilla-La Mancha y Aragón ${ }^{4}$.

La distribución de la producción de energía eólica por comunidades autónomas en España responde también a una gran variedad de problemas y especificidades locales en su implantación y su aceptación social. La sociedad española tiene una percepción de las energías renovables (incluyendo la eólica) muy positiva, y es posible encontrar experiencias modélicas de planificación, implantación, selección de ubicaciones, y participación local. Este es el caso, por ejemplo, de los Planes Energéticos de Navarra, muy cuidadosos con los aspectos sociales y ambientales aunque, asi y todo, no exentos de polémicas focalizadas (Barcos, 2004). También se pueden identificar éxitos locales en la construcción de parques eólicos en algunos municipios de Aragón o Castilla-La Mancha, en los que la energía eólica ha contribuido a dinamizar su economía, no sólo por la venta o alquiler de terrenos, o creando puestos de trabajo en el mantenimiento del parque, sino también con la creación y fortalecimiento de una industria de componentes para aerogeneradores. No obstante, también se encuentran a menudo conflictos a diferentes niveles: relacionados con lo que se entiende como una planificación autonómica insostenible y orientada únicamente al beneficio económico de las grandes compañias eléctricas (por ejemplo, Galicia), o con una ubicación que se percibe como inadecuada por pasar por alto algún valor ecológico o cultural (por ejemplo, los conflictos de la Muela de Carcelén y la Sierra del Boquerón en

ARBOR CLXXXI 715 SEPTIEMBRE-OCTUBRE (2005) 377-392 ISSN: 0210-1963 
Albacete). Por último, encontramos casos de proyectos eólicos novedosos, como los parques marítimos, aún en fase de preparación y en los que a los actores habituales se suman nuevos intereses, como los del turismo o la pesca (González Garcia y Estévez Cedeño, 2004).

La energía eólica se presenta así como un caso de estudio interesante para analizar la relación e implicación del público en las intervenciones tecnocientíficas con efectos sobre el medio ambiente, ya que concita un generalizado apoyo al tiempo que protagoniza serias polémicas.

\section{La energía eólica y el público}

La aceptación pública es uno de los requisitos más importantes para el éxito de los proyectos eólicos ${ }^{5}$. Por ello, desde los comienzos del boom de la energía eólica a mediados de los ochenta, se han llevado a cabo multitud de estudios acerca de la percepción pública de esta nueva forma de energía en expansión. El desarrollo de fuentes de energía renovables, baratas, limpias y descentralizadas es una demanda antigua que puede rastrearse en el pasado reciente al menos hasta el movimiento por una «tecnología alternativa» de los años 70. En 1973, David Dickson defendia formas alternativas de energía, incluyendo la eólica; y en el mismo año Schumacher hablaba de proyectos de uso de "energías intermedias». En ambos casos se reflejaba en sus escritos el estado inicial del desarrollo de la tecnología de aprovechamiento del viento que luego despegaria de forma impresionante. Las grandes organizaciones ecologistas, como Greenpeace, han estado siempre también a favor de las energias renovables, liderando campañas en contra de la alternativa nuclear y a favor de que los gobiernos adopten posiciones claras de apoyo a las energías limpias ${ }^{6}$. El empeño en la promoción de las energías renovables de ecologistas y grupos preocupados por los problemas ambientales derivados del uso de energías no renovables (calentamiento global, agotamiento de los recursos...) está hoy en día en condiciones de hacerse realidad. Es de presagiar, pues, que la percepción pública de la energía eólica sea abiertamente positiva y se den la bienvenida a todas las iniciativas para su promoción.

Sin embargo, el panorama parece ser más complejo. Krohn y Damborg (1999) presentan un análisis de los estudios hechos hasta el momento en diferentes paises acerca de las actitudes públicas hacia la energía eólica. Sus resultados, en general, indican que el público mantiene una idea muy positiva de la energía eólica y se manifiesta a favor de su promoción. Encuestas realizadas en Estados Unidos, Dinamarca o Gran Bretaña arrojan resultados de alrededor de un $80 \%$ de la población a favor de la energía eólica. Sin embargo, cuando se trata de proyectos concretos, el grado de oposición crece considerablemente, condenando al fracaso a algunos de ellos. Adaptando la clasificación que hacen Krohn y Damborg (1999) citando a Simon (1996), y completándola con el estudio del caso español, los argumentos habituales de quienes se manifiestan a favor y en contra de la promoción de la energía eólica se indican en el cuadro 17:

CUADRO 1. Argumentos a favor y en contra de la energía eólica

\begin{tabular}{|l|l|}
\hline \multicolumn{1}{|c|}{ A favor } & \\
\hline - económicamente rentable & \\
\hline - no contaminante & - incapaz de sustituir otras fuentes \\
\hline - renovable & - poco fiable \\
\hline - ayuda a combatir el cambio climático & - cara \\
\hline - segura & - agresiva con el paisaje \\
\hline - creación de puestos de trabajo & - ruidosa \\
\hline - beneficios económicos para los emplazamientos & - perjudicial para las aves \\
\hline & - perjudicial para el medioambiente: desmontes, lineas \\
\hline & - pe evacuación... \\
\hline & - perjudicial para yacimientos arqueológicos \\
\hline & - perjudicial para caladeros de pesca (en el caso de \\
\hline & parques maritimos) \\
\hline
\end{tabular}


Por un lado, la energía eólica tiene ventajas ambientales y económicas, ya que (además de su contribución a la generación de energía renovable) ayuda a mejorar la situación económica de las zonas en las que se implantan parques eólicos (creación de puestos de trabajo en el mantenimiento de los parques y en la industria de fabricación de componentes para aerogeneradores...). Como inconvenientes, pueden señalarse las dudas sobre su capacidad para sustituir las fuentes de energía convencionales y resolver asi los problemas energéticos, los efectos sobre el medio ambiente, el paisaje, la fauna, el problema del ruido de los aerogeneradores para la población cercana y la posibilidad de su interferencia con factores económicos o culturales caracteristicos de las zonas en las que se implanta.

No obstante, el tipo de oposición pública más habitual a la energía eólica no consiste en el rechazo global a esa forma de producción energética, sino más bien en la resistencia a la implementación de un proyecto eólico concreto entre aquellos que sienten que pueden estar afectados por sus consecuencias. Este tipo de resistencia se conoce como NIMBY (Not in My Backyard) ${ }^{8}$, refiriéndose a la oposición del público a la ubicación en la cercanía de sus hogares de instalaciones como vertederos, centrales nucleares, autopistas, centros de salud mental..., aun con el convencimiento general de que se trata de necesidades sociales. Algunos estudios muestran que la oposición a proyectos concretos por parte de los ciudadanos involucrados disminuye una vez que el parque eólico está en funcionamiento (Andersen et al., 1997; Gipe 1995; Bishop y Proctor 1994) y el fenómeno NIMBY parece también disminuir con la información y el conocimiento, ya que es menos común en aquellas zonas en las que ya hay instalados parques eólicos. No obstante, otros estudios proporcionan resultados contradictorios. Thayer $y$ Freeman (1987) analizaron las actitudes públicas ante el desarrollo eólico en la costa californiana, encontrando que aquellos encuestados cuyas viviendas estaban más cerca de los aerogeneradores manifestaban actitudes más negativas que los más alejados, y también que la mayor información, mayor oposición. Kaldellis (2005), por su parte, informa de la ambigüedad de los ciudadanos griegos ante la proliferación de parques eólicos, encontrándose la opinión pública dividida entre los reticentes habitantes de la peninsula y los más positivos habitantes de las islas.

Las diferencias encontradas en los estudios de caso aparecen como indicios de que el factor NIMBY puede ser en muchos casos una explicación demasiado simplista acerca de la oposición pública a la instalación de aerogeneradores (Wolsink, 1988, 2000; Erp, 1997), y que el concepto mismo es confuso. Aducir el fenómeno de NIMBY implica la suposición de que las comunidades locales actúan de un modo egoista, oponiéndose al bien común al tiempo que se oponen a la construcción en sus emplazamientos de un vertedero de productos tóxicos o un centro de salud mental. Los análisis de Wolsink (1994) y Ek (2005) de las actitudes públicas relacionadas con los parques eólicos en Holanda y en Suecia, respectivamente, concluyen que la oposición al emplazamiento local no estaba relacionada con una aceptación general de la energía eólica (ubicando los parques en otro lugar, por supuesto), como revelaría el fenómeno de NIMBY; $y$ que cuando se atiende a las razones para resistir proyectos concretos los factores más relevantes son aquellos que tienen que ver con las características especificas de la ubicación propuesta. De este modo, parecería que la percepción general acerca de las bondades o perjuicios de la energía eólica tiene poco peso en la conformación de estas posiciones de oposición.

Wolsink (2000) distingue cuatro formas de resistencia social frente a proyectos eólicos (que pueden, desde luego, extenderse a la oposición a otro tipo de proyectos, especialmente aquellos relacionados con centrales nucleares y vertederos de residuos tóxicos, temas en los que se ha estudiado más a fondo la oposición del público):

- Tipo A. Actitud positiva hacia la energía eólica, pero negativa hacia la construcción de un parque eólico en las cercanias. NIMBY (Not in My Backyard)

- Tipo B. Actitud negativa hacia la energía eólica y, por tanto, hacia el proyecto en la cercanía de la propia residencia. NIABY (Not in Any Backyard)

- Tipo C. Actitud positiva hacia la energía eólica, que termina siendo negativa como resultado de la discusión durante el proceso de planificación del parque eólico en la cercania de la propia residencia. Esta forma de rechazo indica que las posiciones de los actores de una controversia son dinámicas y cambiantes más que estáticas y predeterminadas, asi como la necesidad de entender los procesos de interacción entre actores y sus efectos.

- Tipo D. Actitud negativa hacia el proyecto en la cercanía de la propia residencia, sin necesidad de rechazar la energía eólica por completo, pero defendiendo su desarrollo bajo ciertos criterios y restricciones. Se basa fundamentalmente en las posibles consecuencias negativas sobre el paisaje, la fauna avicola y el problema del ruido. 
En relación con la energía eólica, el rechazo general de la población es escaso, con lo que el tipo $B$ de actitud es muy limitado. Las actitudes más extendidas e interesantes son la $C$ y la $D$, donde se muestra que el público toma en general decisiones basadas en la racionalización de las ventajas e inconvenientes, y en las que, por otra parte, influyen fuertemente aspectos institucionales relacionados con las políticas de planificación y las acciones de las diferentes partes implicadas. Por ejemplo, elementos de gran relevancia tienen que ver con la confianza depositada en las autoridades y las empresas encargadas del proyecto, es decir, con factores derivados de la interacción entre actores y los intereses locales que se encuentren en conflicto, en línea con los análisis de Brian Wynne (1995) acerca de la complejidad de las actitudes públicas hacia los proyectos tecnocientíficos. Además de evaluar las caracteristicas técnicas del proyecto y sus previsibles efectos económicos, paisajísticos o ecológicos, el público defiende sus posiciones en función de su percepción de cómo los promotores del proyecto y las autoridades locales han planeado e implementado el mismo, y de su experiencia pasada en situaciones análogas. La implicación de la sociedad, la transparencia y la información son todos ellos factores que aumentan la posibilidad de que la población mantenga una actitud positiva ante un proyecto dado (Hansen et al., 2003) ${ }^{9}$.

En el caso español, la especificidad de los conflictos hace sospechar también que el fenómeno NIMBY no es la causa principal de oposición a la energía eólica, sino que se trata más bien de un rechazo focalizado en puntos criticos de desacuerdo, $y$ que en muchas ocasiones se desarrolla a partir de una experiencia previa negativa 0 al cabo de un tiempo de convivencia con un parque eólico que en principio se había recibido con entusiasmo (M. González y B. Estévez, 2004). El proyecto de parque eólico marítimo en la provincia de Cádiz servirá para analizar las percepciones y actitudes públicas, asi como el valor de los mecanismos de participación desarrollados en este caso por la administración en respuesta a la fuerte oposición social.

\section{Energía eólica en Andalucía}

En los inicios de la energía eólica en España, Andalucía ocupó un puesto principal y pionero en su desarrollo, debido a su enorme potencial eólico, fundamentalmente en la provincia de Cádiz. De hecho, el primer parque eólico español se instaló en Tarifa en 1983, y sentó un importante precedente pese a las criticas que posteriormente se realizaron sobre su escasa planificación y los perjuicios ambientales ocasionados
(Sena Rodríguez, 1991). Aves muertas ${ }^{10}$, el riesgo de rotura de aspas (sucedió en 1995) por la fuerza del viento o el impacto paisajístico fueron algunos de los puntos de la polémica sobre este primer parque eólico español. Por diversos motivos el desarrollo inicial andaluz se frenó en los años sucesivos, pasando de ser la primera comunidad productora de energía eólica hace sólo 6 años a ocupar el séptimo puesto en la actualidad. Las causas que se aducen para este retraso andaluz son la insuficiencia de las redes eléctricas y centros de transformación actuales (que están diseñados para la distribución a zonas rurales) para la evacuación de potencia en las comarcas de mayor recurso; asi como la cautela en la concesión de autorizaciones administrativas a los promotores de parques eólicos, ante los conflictos que podrian surgir con otros intereses, tales como la conservación del medio natural. La opinión pública negativa frente a la desordenada planificación eólica en Tarifa ha tenido sin duda influencia en la ralentización eólica en Andalucia. Sin embargo, el actual PLEAN (Plan Energético de Andalucia, 2003-2006) prevé que el $15 \%$ de la energía total demandada por los andaluces tenga su origen en fuentes renovables en 2010, intentando alcanzar en el año 2006 ya una cifra de un 10,6\% (PLEAN, 2003). Las principales provincias donde se desarrollará este plan serán Almería, Málaga y Cádiz.

\section{Participación pública en la gestión eólica gaditana}

La evaluación para la construcción de parques eólicos en Cádiz es realizada principalmente por la Consejería de Innovación, Ciencia y Empresa, la Consejeria de Medioambiente, y sus respectivas delegaciones provinciales. Es notorio que, a pesar de que la instalación de parques eólicos en la provincia data de principios de los años 90, la normativa autonómica sobre los mismos no entra en vigor hasta 1994, con la Ley 794 de Protección Ambiental y el Reglamento de Evaluación de Impacto Ambiental (Decreto 292/95).

Para la puesta en marcha de los primeros parques no existían normativas particulares diseñadas para la evaluación de emplazamientos eólicos, pero los procesos realizados fueron aprobados por las unidades administrativas que hoy dia cumplen las tareas de valoración de los trámites respectivos. En los inicios, la Delegación de Medio Ambiente gaditana cumplió un papel protagonista por dos razones fundamentales: 1) por ser la unidad administrativa directamente implicada y, 2) porque los primeros parques eólicos fueron ubicados en un parque natural de la provincia. 
El seguimiento de la legislación vigente contempla la información a diferentes actores o colectivos implicados o afectados durante cualquier etapa de implantación de un parque eólico. Tal como muestra el proceso reglamentario (Figura 1), se trata de un trabajo llevado a cabo principalmente por las dos unidades administrativas mencionadas (Consejeria de Innovación, Ciencia y Empresa y Consejeria de Medioambiente), y más particularmente por sus delegaciones provinciales. La participación de colectivos sociales tiene lugar en un segundo nivel, es decir, a partir de la evaluación hecha por la administración y de la publicación de propuestas para su evaluación pública. La característica principal de este tipo de participación es que depende de la medida en que tales colectivos se sientan vulnerables ante la propuesta realizada, en otras palabras, el pronunciamiento de la ciudadania se hace cuando se presentan altas probabilidades de generar controversia expresadas a partir de la presentación de alegaciones.
La implicación de la ciudadanía en un segundo nivel (de consulta) es acertada, pero a menudo insuficiente. En el caso de la comarca gaditana de La Janda, la demanda de participación resultó en la aprobación en marzo de 2004 de la propuesta del Plan de Ordenación Territorial de la Janda, que abarca los municipios de Conil de la Frontera, Barbate y Vejér de la Frontera. El Plan de Ordenación Eólico Urbanistico (gestionado por la Consejería de Obras Públicas y Urbanismo) fue elaborado con la finalidad de institucionalizar dónde se pueden instalar plantas eólicas, dónde se pueden instalar pero corrigiendo una serie de reparos y dónde está completamente prohibido construir plantas eólicas. Dicho plan fue producto de foros de participación en los que intervinieron Ecologistas en Acción como representante de las organizaciones sociales y demás agentes interesados en la temática. El resultado de consenso parece haber generado un plan bastante acotado y aceptable por todos los actores sociales involucrados.

Figura 1. Procedimiento de toma de decisiones sobre parques eólicos en Cádiz

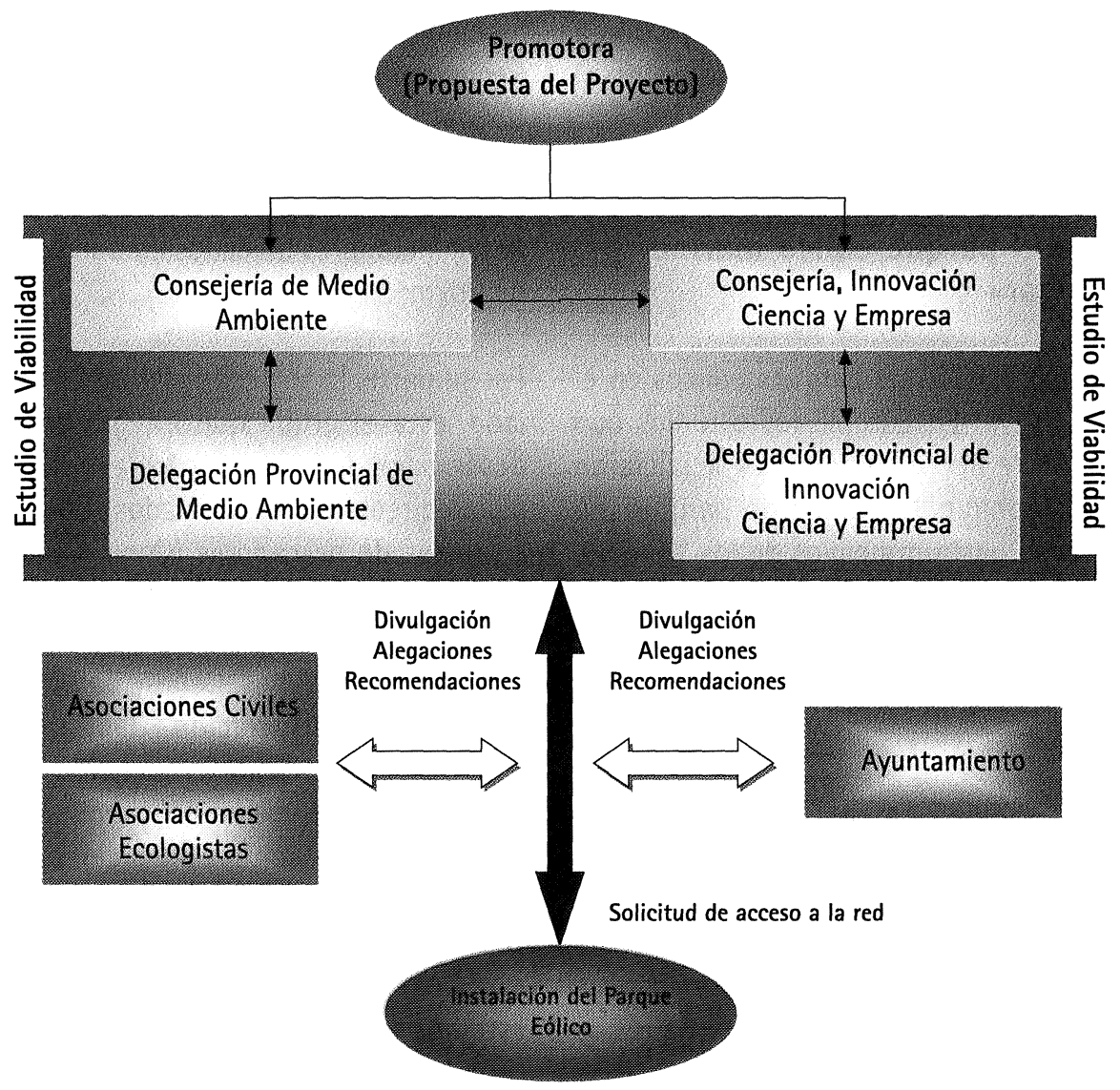


Sin embargo, uno de los proyectos especialmente conflictivos es el del desarrollo eólico marino planeado en la costa del cabo de Trafalgar, en Cádiz. Los parques marítimos, en los que los aerogeneradores se colocan mar adentro, son una apuesta de futuro en el campo de la energía eólica, aunque en el plan energético español no están aún contemplados y la normativa existente no recoge sus especificidades, como por ejemplo, que su construcción es mucho más costosa que la de un parque eólico terrestre. El caso del parque maritimo de Trafalgar revela una peculiar distribución de las voces a favor y en contra. Mientras los grandes grupos ecologistas como Greenpeace, Ecologistas en Acción o Los Verdes de Andalucía se manifiestan a favor del desarrollo de parques eólicos marítimos en general, y en concreto, de este de Trafalgar, aduciendo el escaso impacto ambiental del mismo; fueron los ayuntamientos, los pequeños grupos conservacionistas locales, las cofradias de pescadores y el sector turístico de la zona los que se opusieron con más énfasis a la creación del parque sobre la base de sus perjuicios paisajísticos y los efectos sobre el ecosistema marino, la pesca y la navegación ${ }^{11}$. La provincia de Cádiz aparece así como un interesante caso de estudio, en el que se une una historia previa de mala gestión a unos proyectos de futuro que plantean nuevos retos al afectar a más actores sociales.

\section{El camino hacia el parque eólico marino "Cabo de Trafalgar"}

La historia sobre la implantación de parques eólicos en el mar es relativamente nueva. En Europa, los estudios que permitieron desarrollar este tipo de propuestas se iniciaron en los años 70 y 80 , aunque los primeros parques no comenzaron a construirse hasta principios de los años 90 . Actualmente, existen parques eólicos marítimos construidos y proyectados en paises como Alemania, Suecia, Dinamarca, los Paises Bajos, Irlanda y el Reino Unido (BWEA, 2004).

En el caso español, las condiciones del suelo submarino para este tipo de industria no son especialmente adecuadas, pero destacan algunas zonas: el Golfo de Cádiz, por la calidad del aire, el gran potencial eólico y la favorable situación oceanográfica; la costa del Mediterráneo, por sus fondos no muy profundos. Otras zonas, como Galicia y algunos puertos, permiten emplazamientos singulares. Pese existen factores técnicos y económicos que motivan el interés de empresas promotoras y el apoyo de parte de la administración. En cuanto a los factores técnicos, la energía eólica en el mar permite mejorar el rendimiento de las ubicaciones terrestres, al introducir importantes innovaciones tecnológicas en los aerogeneradores que resultan en una producción eléctrica superior a la que puede ser obtenida en tierra, y además estas instalaciones pueden ir conectadas directamente a la línea de distribución. Los parques eólicos en el mar resultan asi rentables por su nivel de producción energética (Menéndez, 2005).

El actual avance de la energía eólica en la provincia de Cádiz se focaliza en el proyecto de parque eólico marino frente al Cabo de Trafalgar y, más especificamente, en las costas de los pueblos pesqueros Conil de la Frontera y Barbate. La primera solicitud se llevó a cabo por parte de EHN en el año 2000; desde entonces, los procesos de tramitación se han sucedido en una constante de cumplimientos normativos, intercambios entre los diferentes agentes involucrados, presentación de alegaciones, resoluciones, exposición de nuevas propuestas de proyectos, evaluaciones, declaraciones y propuestas de negociaciones. Recientemente, cuatro promotores (EHN, Capital Energy, Umweltkontor y Vestas Eólica) han solicitado los permisos correspondientes para la instalación de parques eólicos marinos. En conjunto se trataria de un total de más de 500 aerogeneradores ubicados en una extensión marina de más de $20 \mathrm{~km}^{2}$.

Por su parte, la sociedad civil se encuentra dividida. Por un lado, está el grupo que integra una plataforma formada desde hace dos años por los ayuntamientos de tres municipios directamente afectados (Conil, Vejér y Barbate), las cofradias de pescadores (Conil y Barbate), representantes de cuatro grupos políticos (PP, PSOE, IU, PA), sindicatos $\left(\mathrm{CCOO}\right.$ y UGT) ${ }^{12}$, cuatro grupos de almadraberos (Tarifa, Zahara de los Atunes, Conil y Barbate) y el grupo ecologista AGADEN (Asociación Gaditana en Defensa de la Naturaleza). Esta plataforma comparte una visión desfavorable a la construcción del parque eólico marítimo, por lo que han presentado las alegaciones que consideran pertinentes. Por otro lado, los grupos de Ecologistas en Acción, Greenpeace y Verdes de Andalucia mantienen una actitud más favorable, mostrándose dispuestos a que se hagan los estudios de preinstalación de torres experimentales, lo que serviría para dar un diagnóstico más acertado y objetivo de los posibles efectos en la fauna biomarina de la región. 
Así, mientras que la situación actual del desarrollo eólico sobre plataforma terrestre en Cádiz ha logrado el acuerdo de las partes implicadas y se desarrolla con normalidad, el proyecto de parque eólico marino ha generado una fuerte resistencia social. No se trata entonces de una simple reacción NIMBY, puesto que existe una visión positiva hacia la energía eólica y la resistencia se presenta sólo ante la propuesta concreta de implantación de un parque marino que entra en conflicto con las tradiciones culturales y actividades económicas locales. Esta forma de rechazo indica que las posiciones de los actores de una controversia son dinámicas y contextuales más que estáticas y predeterminadas, así como la necesidad de entender los procesos de interacción entre actores y sus efectos.

Más detalladamente, las razones de la oposición local al parque eólico marino son las siguientes:

- Su interferencia con tradiciones económicas y culturales locales como la pesca de almadraba, con la consiguiente pérdida de los empleos que dependen directa 0 indirectamente de ellas. Las vibraciones producidas por los aerogeneradores provocarian cambios en las rutas migratorias de los atunes, y los barcos de pesca artesanal verían dificultado su trabajo por la ubicación de los mismos.

- Frente al empleo de calidad proporcionado por la pesca tradicional, el empleo ofrecido por el parque eólico es fundamentalmente temporal.

- Hay especies marinas en la zona que aparecen incluidas en el Catálogo de Especies Amenazadas (Orden del 9 de Junio de 1999, BOE del 22.6.99), como la caracola, el puercoespín marino, y la lapa ferruginea.

- El turismo puede verse afectado por el impacto visual de los aerogeneradores.

- El Cabo de Trafalgar es una zona de gran valor histórico y arqueológico. Además de la Batalla de Trafalgar, los fondos marinos de la zona conservan rastros de fenicios, romanos y árabes que forman parte del patrimonio cultural español aún sin explo$\operatorname{rar}^{13}$.

Existen otra serie de razones que tienen que ver con la desconfianza pública hacia los procesos desarrollados desde la administración: el desordenado desarrollo eólico, la incertidumbre creada por algunas empresas que no dialogaron con los agentes locales...

\section{El Foro de la Energía Eólica Marina y el Desarrollo Sostenible}

La situación generada entre los diferentes agentes sociales implicados y afectados por la posible instalación del parque eólico marítimo llegó a tener tanta repercusión pública que el Área de Medioambiente de la Diputación de Cádiz (por solicitud de un representante de uno de los colectivos sociales preocupados por la temática) organizó el «Foro de la Energía Eólica Marina y el Desarrollo Sosteniblen. El Foro surge así como propuesta para encaminar y organizar los procesos de participación pública en la toma de decisiones sobre energía eólica marina en Cádiz. Para la puesta en marcha de dicho instrumento fue necesario un esfuerzo exhaustivo de identificación de actores sociales, considerando las siguientes categorias de participantes: representantes de la administración central, autonómica y local; las empresas promotoras; expertos universitarios; y los diversos agentes sociales implicados, incluyendo grupos de ecologistas, cofradias de pescadores y demás organizaciones con puntos de vistas diversos y pertinentes.

Los objetivos generales del Foro fueron los siguientes:

- identificar y convocar a los agentes implicados e interesados en la temática planteada,

- definir y comunicar las directrices metodológicas del ejercicio a los integrantes,

- crear un ambiente coordinado de diálogo entre los participantes,

- informar con transparencia de los argumentos que justifican la presencia de cada agente o institución representada,

- concienciar a los colaboradores, a los organismos competentes, a los medios de comunicación y a la ciudadania en general sobre la importancia de este tipo de actividades para la resolución de conflictos,

- fomentar la comunicación y el entendimiento entre los participantes,

- mejorar los planteamientos iniciales de la temática que justifica la convocatoria de trabajo,

- sistematizar y clasificar la documentación obtenida,

- servir de catalizador de información para mejorar el proceso de evaluación previo a la toma de decisiones.

El Foro desarrolló sus actividades entre noviembre de 2004 y junio de 2005, con una periodicidad quincenal de reuniones en la Diputación de Cádiz, a las cuales se invitaron a 
53 actores. Hubo 31 intervenciones de los actores y 7 de delegaciones administrativas, con lo que en total la asistencia al foro superó el $70 \%$ de las invitaciones cursadas. Cada reunión contó con un moderador ${ }^{14}$ que presentaba a los ponentes e informaba sobre las novedades en curso, y comenzaba con el reparto a los asistentes de documentación en la que se incluian las noticias aparecidas en la prensa, informes de expertos y las respuestas de los invitados de la sesión a un cuestionario previo sobre el asunto elaborado por la organización del Foro. Cada ponente tenía entre 15 y 20 minutos para exponer sus puntos de vista, posteriormente se abria una ronda de preguntas en las que intervenían los concejales de medioambiente, representantes de diferentes partidos políticos, y demás agentes participantes. Los medios de comunicación local también estaban invitados al cierre de cada reunión como intermediarios entre los participantes en el foro y la sociedad en general. La apertura y mantenimiento de una página web (http://www.foroeolica.dipucadiz.org/) donde figuraba documentación relevante y se recogian todas las noticias aparecidas en la prensa (local, nacional y especializada) sobre el conflicto fue también un pilar básico para mantener a la ciudadania informada de la marcha del Foro y los debates ${ }^{15}$. Ha de destacarse que este novedoso ejercicio despertó durante más de medio año una gran relevancia pública, especialmente en los ámbitos local y autonómico, pero también a nivel nacional e internacional.

Como cierre del Foro se planificaron unas Jornadas (9 y 10 de Junio de 2005) que funcionaron como un instrumento añadido a las reuniones celebradas, la web y el cuestionario elaborado por la Diputación. Las jornadas permitieron una participación aún más amplia de los diferentes agentes que ya habian expresado sus opiniones durante el foro, pero la diferencia es que este tipo de metodología permitió la presencia simultánea de todos ellos: expertos de diferente signo, empresas promotoras, agentes sociales, representantes de la administración e instituciones independientes. La interacción directa de posturas fuertemente encontradas dio lugar a algunos momentos de tensión que potenciaron el interés mediático del evento.

Tras las Jornadas, el Foro Eólico hizo públicas sus conclusiones en julio de 2005. Sin embargo, antes incluso de las mismas, la repercusión pública de la polémica tuvo efectos reseñables: las administraciones central, autonómica y provincial parecieron ponerse de acuerdo en que, por el momento, no era posible construir los parques. La falta de legislación sobre energía eólica marítima, la escasez de estudios concluyentes sobre sus efectos en la zona y la clara oposición popular fueron los factores responsables de las decisiones tomadas.

\section{Gobernanza eólica en Cádiz: una evaluación}

El desarrollo del Foro de la Energía Eólica ha sido ambivalente. A la innegable ventaja de ser capaces de sentar a una misma mesa posiciones opuestas, se unen distintos problemas, como la falta de claridad respecto a las atribuciones del Foro, el carácter no vinculante de sus decisiones, y la desconfianza de algunos de los participantes en el valor de la colaboración.

Una evaluación posible del Foro de la Energía Eólica y el Desarrollo Sostenible de la Diputación de Cádiz como mecanismo de participación social puede realizarse siguiendo los criterios identificados por Rowe y Frewer (2000). Los autores clasifican estos criterios en dos grupos: criterios de aceptación y criterios de procedimiento. Los criterios de aceptación son criterios relacionados con la construcción efectiva y el funcionamiento de los mecanismos:

$>$ Representatividad: el público implicado en el proceso de participación debe ser lo suficientemente representativo. En este caso, el Foro reunió a una amplia representación de los intereses implicados: agrupaciones de ecologistas internacionales, nacionales y locales, alcaldes, sindicatos, expertos de la Universidad, empresas promotoras, asociaciones de pescadores, arqueólogos y representantes de la administración autonómica ${ }^{16}$.

Pese a que España no es un país con gran tradición en asociacionismo y participación, el papel de las organizaciones de ciudadanos adquiere cada vez mayor relevancia. En este caso han participado de forma activa asociaciones ambientalistas de distintos niveles; asi como asociaciones de vecinos, ayuntamientos y asociaciones "gremiales". Mientras que habitualmente se tiende a homogeneizar la percepción u opinión del "público", nos encontramos aquí con que las distintas asociaciones ciudadanas que han contribuido y presionado para promover su participación en el mismo presentan no sólo perfiles muy distintos, sino también actitudes muy diferentes. Incluso dentro de las asocia- 
ciones ambientalistas, mientras las de ámbito más amplio apoyaron el plan de construcción de parques eólicos en el Mar de Trafalgar, no ocurre lo mismo con las de ámbito local, que mostraron una firme oposición.

La organización del Foro, no obstante, permitía únicamente la entrada en el juego de representantes de intereses organizados, no ofreciendo la oportunidad de participación directa a los no-expertos. Las sesiones del Foro no fueron abiertas al público, aunque las Jornadas sí permitieron la asistencia de todo aquel ciudadano interesado (previo pago de la cuota de inscripción).

Independencia e igualdad: se debe garantizar la igualdad de todas las partes, conduciendo el proceso de participación de un modo independiente y sin sesgos. En el caso del Foro de la Energia Eólica Marina de Cádiz, la Diputación permitió la participación equitativa e independiente de todos los involucrados y fue un ente de equilibro para mantener el proceso sin ningún tipo de sesgo. Sin duda, la posición de neutralidad de la Diputación se vio favorecida al no depender de ella el destino final de los proyectos del Mar de Trafalgar.

Implicación activa: en el caso ideal, el público participante debe involucrarse activamente desde las fases iniciales de la definición de problemas, no limitándose su participación a la elección entre una serie de posibilidades determinadas de antemano por expertos. La participación social durante los procesos de implantación de parques eólicos es fundamentalmente, como ya se ha señalado, una participación reactiva (presentación de alegaciones y recursos a proyectos). Dadas las dificultades en los procesos de información y comunicación, este tipo de participación, si bien necesaria, resulta a todas luces insuficiente. No obstante, el Foro Eólico se convoca en un momento apropiado, en la etapa de diagnóstico y planificación del proceso, permitiendo una implicación más activa y la paralización efectiva de los proyectos antes de su puesta en marcha.

Efectividad e influencia: el resultado del procedimiento debe tener un impacto real y efectivo sobre las decisiones adoptadas en todas las fases del proceso, desde la discusión hasta la toma de decisiones y la ejecución de las mismas. El principal problema del Foro de la Energía Eólica Marina fue precisamente la falta de claridad sobre los efectos de sus conclusiones. El Foro fue una iniciativa de la Diputación de Cádiz en la que no se involucró ni el Gobierno Autonómico ni el Gobierno central (del que dependía en última instancia la decisión final). Las conclusiones del Foro no podian, por tanto, ser vinculantes. Al no participar tampoco en el mismo representantes de los niveles autonómico y central, el debate se vio limitado en su capacidad de influencia.

Dransparencia: el desarrollo del proceso y la toma de decisiones deben ser transparentes y comprensibles para el público en general. Los medios de comunicación, especialmente los locales, jugaron un papel clave durante todo el proceso, amplificando su relevancia pública. Además de su repercusión en la prensa local, el conflicto de la eólica marina en Cádiz tuvo eco también en periódicos regionales y locales, así como en algunas publicaciones internacionales relacionadas con las energías alternativas (Korneffel, 2005).

Por otra parte, existen diferentes criterios de procedimiento. Se trata de criterios relacionados con la potencial aceptación pública e institucional del mecanismo o procedimiento.

> Accesibilidad de recursos. El público participante debería tener acceso a los recursos apropiados para el satisfactorio desempeño de su tarea. En este caso, la existencia de la página web con abundante documentación ayudó enormemente a facilitar el acceso a la información a todos aquellos ciudadanos interesados en la polémica.

$>$ Definición de los objetivos. La naturaleza y alcance de los objetivos y actividades de la participación deberian estar definidos con claridad. Aunque los objetivos del Foro eran aparentemente claros, los límites impuestos por las competencias de la Diputación desvirtuaron el proceso. Algunos sectores, como las asociaciones de pescadores y almadraberos y las asociaciones ambientales locales desconfiaban profundamente de la utilidad del Foro. Su sospecha es que sólo serviría de pantalla para justificar una decisión tomada de antemano. Crear relaciones de confianza entre los distintos agentes sociales, y en especial con la administración como intermediaria entre los mismos aparece de nuevo como un problema central a resolver en las iniciativas de gobernanza.

D Estructuración de la toma de decisiones. El ejercicio de la participación debería hacer uso de los mecanismos 
apropiados para estructurar con claridad el proceso de toma de decisiones. La inexistencia en España de regulaciones sobre parques eólicos marinos crea un vacio legal y da lugar a la improvisación y a la precipitación en el caso de los proyectos eólicos marinos en la costa de Trafalgar. La falta de estudios claros de impacto sobre el ambiente, la pesca, el turismo, los yacimientos arqueológicos..., y de una legislación nacional sobre este tipo de instalaciones, parece haber terminado por cerrar, al menos provisionalmente, el conflicto. De hecho, la clausura del conflicto tras el anuncio en la prensa, antes de la publicación de las conclusiones del Foro, de que tanto el Gobierno Autonómico como el Central desestimaban la construcción del parque eólico, supuso de algún modo una deslegitimación del mismo, aun cuando el rechazo respondió a la oposición social, a la falta de legislación sobre aprovechamiento eólico marino y a las incertidumbres sobre sus impactos.

$>$ Relación coste/efectividad. El procedimiento deberia ser efectivo con relación al coste. Creemos que fue adecuado en este punto.

En definitiva, el Área de Medio Ambiente de la Diputación de Cádiz ha respondido adecuadamente al conflicto social originado por la propuesta de parque eólico con la creación del Foro de la Energía Eólica Marina y el Desarrollo Sostenible. La iniciativa de crear un espacio para la presentación de las diferentes posturas, el intercambio de informaciones y opiniones, la discusión y la evaluación es una auténtica acción de gobernanza en la que se han implicado expertos, políticos y representantes de la ciudadanía. La implicación en el Foro de diferentes niveles de la administración ha permitido también identificar y explicitar las distintas percepciones, opiniones y sensibilidades que también existen en la misma. Las Jornadas Técnicas, a su vez, han ayudado además a promover la interacción cara a cara de las diferentes posturas; estrategia que, aunque arriesgada cuando existen importantes tensiones como en este caso, puede permitir buenos resultados en otros al ofrecer oportunidades para la negociación.

Sin embargo, para unos resultados óptimos, el Foro deberia haberse enmarcado en una estrategia conjunta de los distintos niveles administrativos para la toma de decisiones, aclarando las respectivas competencias y responsabilidades. La clausura de este caso, en la que no queda clara la influencia dèl Foro ya que no se ha esperado a sus con- clusiones (aunque si parezca clara la influencia del conflicto social desarrollado alrededor del mismo), resta relevancia a la iniciativa de participación.

\section{Consideraciones finales}

El aprovechamiento eólico en la provincia de Cádiz ha estado dirigido por la implantación de los parques eólicos propuesta por los promotores antes de la definición de normativas, situación que se presenta como denominador común en buena parte del territorio español. En el caso del proyecto de energía eólica maritima en el Mar de Trafalgar, esta carencia de normativa fue uno de los obstáculos principales con los que se encontró la iniciativa de participación organizada por la Diputación de Cádiz.

En efecto, el plan de desarrollo eólico marino en Cádiz originó una importante protesta social y dio lugar a un amplio debate en el que la necesidad de producción energética de fuentes alternativas chocaba frontalmente con los intereses y percepciones de las comunidades locales. No puede entenderse, sin embargo, la fuerte oposición al proyecto como una reacción NIMBY, al relacionarse con una aceptación general de las energias alternativas; con preocupaciones concretas y razonables sobre los diversos efectos ambientales, económicos y culturales de la construcción de los parques en la zona; y con la percepción acertada de la falta de conocimientos, normativas y planificaciones suficientes para llevar a cabo el proyecto minimizando sus desventajas.

A la vista de la polémica originada, la Diputación de Cádiz reaccionó con oportunidad y acierto ofreciendo un espacio para el diálogo y el intercambio de opiniones. La iniciativa del Foro Eólico y las Jornadas Técnicas fue, por tanto, muy positiva, aunque se enfrentó a algunas limitaciones.

El principal escollo lo constituyó la ausencia de normativa para la instalación de parques eólicos marinos en España, lo que hace que la aprobación de dicha instalación sea competencia directa de representantes de la Administración Central, especificamente del Ministerio de Medio Ambiente y el Ministerio de Industria, conjuntamente con la Junta y las Consejerias respectivas. Ante esta situación, la Diputación de Cádiz siempre manifestó a los participante del Foro que los resultados generados en el mismo no serian vinculantes respecto a la decisión defini- 
tiva, que no estaba en su mano. Siguiendo esta misma línea, la alusión a la ausencia de representantes de entes administrativos competentes fue otra de las principales constantes durante el desarrollo de las Jornadas, resaltando que en un proceso común y objetivo la toma de decisiones no puede hacerse a espaldas o sin escuchar a los actores directamente implicados o afectados.

Otra dificultad detectada, y ligada a la anterior, ha sido el escepticismo con el que la mayor parte de los grupos que forman la Plataforma de rechazo al proyecto eólico vieron tanto el Foro como las Jornadas. Representantes de ayuntamientos y cofradias de pescadores dudaron hasta el último momento acerca de su participación, desconfiando de su capacidad de modificar una decisión que creían tomada de antemano. El hecho de que fuesen organizadas por la administración creó cierta desconfianza, que puede estar fundada en experiencias previas de intentos de instrumentalizar la participación, utilizando los mecanismos de deliberación social para legitimar decisiones previas e inamovibles. Al no haber ningún compromiso político ligado a las conclusiones del Foro, unido a la percepción de que fue la trascendencia pública de la polémica a través de los medios de comunicación lo que motivó finalmente la paralización provisional del proyecto de desarrollo eólico (más que la efectividad misma del Foro y las Jornadas), la relevancia de los detalles específicos del mecanismo de deliberación elegido parece difuminarse. De hecho, el cierre de la disputa, con la paralización provisional del proyecto, anunciado en los medios de comunicación antes incluso de la publicación de las conclusiones del Foro resta en cierto modo legitimidad al mismo. En última instancia, pareceria que el método más efectivo de participación social es el de la protesta y su difusión a través de los medios, más que el de la negociación formal propiciada desde la administración. Sin embargo, consideramos que, en este caso, la trascendencia pública de la polémica y el mecanismo formal promovido desde la administración fueron estrategias que se apoyaron mutuamente para paralizar provisionalmente el proyecto. La protesta social fue determinante para que la administración convocara el Foro, que a su vez, mantuvo vivo el debate, la generación de argumentos y su repercusión en la prensa durante los meses que duraron sus sesiones. Participación formal y espontánea se complementaron de este modo haciendo visibles los intereses de los afectados.

Por último, es de señalar que el caso del proyecto del parque eólico marino Cabo de Trafalgar plantea también la conveniencia de revisar la normativa vigente para la instalación de parques eólicos en tierra, que permite la participación ciudadana sólo en un segundo nivel, es decir, en la presentación de alegaciones, ya que existe una clara demanda para que aumente el nivel de participación ex ante de los directamente implicados, en las etapas del diseño de las intervenciones. 
1 Este trabajo ha sido posible gracias al estudio llevado a cabo bajo el convenio FECYT/IFS-CSIC sobre "Sociedad civil y gobernanza de la ciencia y la tecnologia en Españan. Agradecemos especialmente la colaboración de todas las personas entrevistadas para su realización.

2 Este es el caso de la reciente ratificación del Convenio de Aarhus sobre el acceso a la información, la participación del público en la toma de decisiones y el acceso a la justicia en materia de medio ambiente (firmado en 1998 y que entró en vigor en España el 29 de marzo de 2005).

3 http://www.windenergy.org.nz/documents/2005/050304-GWEC-Global2004 WindStats.pdf

4 Las Comunidades Autónomas sin desarroIlo eólico son Cantabria, Extremadura y Madrid. En algunos casos por la reticencia de sus gobiernos y en otros porque no se dan buenas condiciones para la explotación de los recursos eólicos.

5 En el informe del Congreso sobre "Renewable Energy - Intelligent Policy Optionss, organizado en Bonn en enero de 2004 por la Comisión Europea y EREC (European Renewable Energy Council) se reconocía que: "la falta de información y concienciación es aún una de las barreras más importantes frente a la utilización de energías renovables. Las políticas de comunicación e información son esenciales para incrementar la sensibilización pública y asegurar el apoyo público y privado. Un enfoque combinado para promover la eficacia energética y las fuentes renovables junto a medidas de diseminación bien definidas es deseable para promocionar el desarrollo de las energías renovables" (véanse las conclusiones del congreso y sus recomendaciones en: http://www.eufores.org/Summary.htm).

6 http://archivo.greenpeace.org/energia/ index.htm

7 Thayer y Freeman (1987) y Wolsink (2000) proporcionan una lista de razones similar.

8 "No en mi patio trasero».
9 La legislación europea sobre parques eólicos ha incorporado recientemente una Directiva sobre SEA (Strategic Environmental Assessment - Evaluación Ambiental Estratégica, Directiva 2001/42/EC), que tiene como objeto mejorar los canales de información y comunicación entre promotores de los proyectos y el público, e involucrar a la población afectada en la elaboración de los proyectos en lugar de esperar sus reacciones a proyectos ya cerrados. Las evaluaciones estratégicas de impacto ambiental constituyen, sin duda, un paso adelante respecto a las evaluaciones de impacto ambiental convencionales, en lo que respecta a las posibilidades del público de influir sobre los planes; aunque, lo mismo que estas, su éxito dependerá en parte de la voluntad de las partes implicadas en favorecer esta participación.

10 Windpower Monthly, 2 de febrero de 1994.
11 En el contexto del Programa ALTENER 2001 (2002) de la Comisión Europea para el desarrollo de energias alternativas, se realizó un estudio sobre aceptación pública de parques eólicos marítimos en España y Portugal. Las zonas del Delta del Ebro y el Estrecho de Gibraltar fueron las elegidas en el caso de España por ser las más apropiadas para la instalación de estos parques. Uno de los objetivos del proyecto ALTENER fue el de analizar los obstáculos ambientales, políticos y sociales a los que podrian enfrentarse los proyectos de parques eólicos marítimos, una alternativa que ya está funcionando con éxito en Gran Bretaña, Suecia, Dinamarca, Holanda y Alemania. El caso de la Peninsula Ibérica es especialmente delicado en este sentido, se reconoce, debido a la intensa actividad económica ligada al turismo, la pesca y el tráfico maritimo. Las encuestas realizadas, tanto a residentes como a turistas, dieron como resultado un considerable apoyo a la instalación de parques eólicos marítimos (42\%) frente a un $26 \%$ en contra. Las tendencias generales eran a favor de las energías alternativas $(96 \%)$ y de confianza hacia los grupos ecologistas (76\%). Lo que preocupaba a los encuestados respecto a los parques eólicos marítimos era fundamentalmente los efectos negativos sobre aves y peces $(47 \%)$, el paisaje $(22 \%)$, los accidentes marítimos (13\%), la pesca (7\%) y, en menor medida, el turismo (3\%). Las ventajas, sin embargo, las cifraban en la limpieza (65\%), la producción de energia $(15 \%)$, la reducción de consumo de combustibles no renovables (13\%), las inversiones (3\%), el interés añadido al paisaje $(1 \%)$ y el atractivo turístico (1\%). Se identificaron diferencias entre residentes y turistas, de tal modo que los residentes estaban en general menos a favor de la instalación de los parques que los turistas, mostrando mayor preocupación por la afectación a las formas de vida locales: pesca, navegación, turismo... Por contra, las mayores preocupaciones de los turistas, en general más favorables a la implantación, eran los efectos sobre el paisaje y las aves (Un 11\% de los turistas manifestaron que no volverian a la zona tras la instalación del parque maritimo). 
12 En el caso de los partidos políticos y sindicatos, sólo los representantes locales forman parte de la plataforma, ya que a nivel autonómico y nacional, las visiones de estos grupos son encontradas y algunas se mantienen en reserva con respecto al parque eólico marino Cabo de Trafalgar.

13 Este aspecto pone en relieve otras necesidades de normativas, pues el Reglamento de Protección del Patrimonio no regula los espacios subacuáticos, según manifestó Milagros Alzaga en su intervención en el Foro de la Energía Eólica en representación del Centro Andaluz de Arqueologia Subacuática perteneciente a la Consejeria de Cultura de la Junta de Andalucia.

14 Papel que fue desempeñado por el Vicepresidente Primero, Diputado Delegado de Medioambiente de la provincia de Cádiz, Sr. Manuel Maria De Bernardo Foncubierta.

15 En la página web se incluyó también un foro de participación público, que registró poca actividad.

$16 \mathrm{El}$ único representante de la administración autonómica participó como miembro que forma parte de la directiva de la asociación ecologista Verdes de Andalucia.

\section{BIBLIOGRAFÍA}

ALTERNER (2001): Methodological

Guidelines for the Environmental and Socioeconomic Impact Assessment of Off-Shore WindFarms in Touristic Areas.

Project Contract N 4. 1030/Z/01036/2001.

Álvarez-Farizo, B. \& N. Hanley (2002): Using Conjoint Analysis to Quantify Public Preferences over the Environmental Impacts of Wind Farms. An Example from Spain, Energy Policy, 30, 107-116.

Banthien H, M. Jaspers \& A. Renner (2003): Governance of the European Research Area. The role of civil society, European Commission. Community Research, IFOK Interim Report.

<http:/europa.eu.int/comm/research/ sciense-society/>.

Barcos, R. (2004): Desarrollo eólico en la Comunidad Foral de Navarra, Documento de trabajo IF/FECYT. Madrid.

Bishop, K. \& A. Proctor (1994): Love Them or Loathe Them? Public Attitudes Towards Wind Farms in Wales, Cardiff. Cardiff School of City and Regional Planning, Papers in Environmental Planning Research 8.

Brown, L.R. (2004): Europe Leading World Into Age of Wind Energy, Eargh Policy Institute. http:/www.eart-policy.org/ Updates/Update37.htm

BWA (2004), Prospects For Offshore Wind Energy, Informe/Altener XVII/4.1030/Z/98-395.

Cavallaro, F. \& L. Ciraolo (2005): A Multicriteria Approach to Evaluate Wind Energy Plants on an Italian Island, Energy Policy, 33/2: 235-244.

Comisión Económica para Europa/Comité de Política Ambiental (1999):

Convención sobre el acceso a la información, la participación del público en la toma de decisiones y el acceso a la justicia en asuntos ambientales (Convención Aarhus), ECE/CEP/ INFORMAL/1999/1.

Chopyak J \& P. Levesque (2002): Public participation in science and technology decision making: trends for the future. Technology in Society, 24, 155-166.

Delas, M. (2003): El entorno normativo de la energía eólica, Energía, octubre 2003.
Ek, K. (2005): Public and Private Attittudes Towards 'Green' Electricity: The Case of Swedish Wind Power, Energy Policy, 33/13: 1677-1689.

Erp, F. (1997): Sitting Processes for Wind Energy Projects in Germany, Eindhoven University of Technology

EWEA (2003): Wind Energy: The Facts. Analysis of Wind Energy in the EU-25 http:/www.ewea.org/06projecsts_events/ proj_WEfacts.htm

Fiorino, D. (1990): Citizen Participation and Enviromental Risk: A Survey of Institutional Mechanisms, Science, Technology and Human Values, 15/2, 226-243.

González Garcia, M.I \& B. Estévez Cedeño (2004): Energía eólica: conflictos sociales en la planificación y emplazamiento, Documento de trabajo IF/FECYT. Madrid.

Gipe, P. (1995): Wind Energy Comes of Age, Nueva York: Wiley \& sons.

Hanley, N. \& C. NEVIN (1999): Appraising Renewable Energy Developments in Remote Communities: The Case of the North Assynt Estate, Scotland, Energy Policy, 27, 527-547.

Hansen, L.K., K. Hammarlund, H.C. Sorensen \& L. Christensen (2003): Public Acceptance of Wave Energy, Proceedings from the $5^{\text {th }}$ European Wave Energy Confrence, Dublin: University College.

Hidalgo, C. (2005): Experiencia internacional en el sector de la Energía Eólica Marina. Ponencia presentada en las Jornadas sobre energía eólica marina y desarrollo sostenible en la Provincia de Cádiz. Junio 9-10.

Kabouris, J. \& K. Perrakis (2000): Wind Electricity in Greece: Recent Developments, Problems and Prospects, Renewable Energy, 21, 417-432.

Kaldellis, J.K. (2005): Social Attitudes Towards Wind Energy Application in Greece, Energy Policy, 33/5: 595-602.

Korneffel, P. (2005): A new battle of Trafalgar, New Energy. Magazine for renewable energy, 1/enero.

Krohn, S. \& S. Damborg (1999): On Public Attitudes Towards Wind Power, Renewable Energy, 16, 954-960. 
López Cerezo, J.A. \& González Garcia, M.I. (2002): Politicas del bosque: expertos políticos y ciudadanos en la polémica del eucalipto en Asturias, Madrid:

Cambridge University Press/OEl.

López Cerezo, J.A., J.A. Menéndez Sanz \& 0. Todt (1998): Participación pública en política tecnológica: problemas y perspectivas, Arbor, CLIX/627, 279-308.

Martínez Camarero, C. (1999): Energías renovables. Plan de fomento de las energías renovables, Daphnia, Boletín informativo sobre la prevención de la contaminación y la producción limpia, 21.

Menéndez, E. (2005): Condicionantes técnicos y ambientales en la eólica marina, Ponencia presentada en las Jornadas sobre energía eólica marina y desarrollo sostenible en la Provincia de Cádiz. Junio 9-10.

PLEAN (2003): Plan Energético de Andalucía, Consejeria de Innovación, Ciencia y Empresa.

Rowe, G. \& L. Frewer (2000): Public Participation Methods: A Framework for Evaluation, Science, Technology and Human Values, 25/1: 3-29.

Sena Rodriguez, I. (1991): El futuro prometedor de la energía eólica en Tarifa, Aljaranda. Revista de Estudios Tarifeños, año I, nº 0.

Thayer, R.L. \& C.M. Freeman (1987): Altamont: Public Perceptions of a Wind Energy Landscape, Landscape and Urban Planning, 14: 379-398.

Todt, 0. (2003a): Potencialidades y riesgos de la participación, en: López Cerezo J \& Ibarra A. (eds): La democratización de la ciencia. San Sebastián: Cátedra Sánchez-Mazas.

Todt, 0. (2003b): Designing trust, Futures, 35: 239-251.

Walker, G. (1995): Renewable Energy and the Public, Land Use Policy, 12/1: 49-59.

Wolsink, M. (1988): The Social Impact of a Large Wind Turbine, Environmental Impact Assessment Review, 32/8(4): 324-325.

Wolsink, M. (1996): Dutch Wind Power Policy, Energy Policy, 24/12: 1079-1088.

Wolsink, M. (2000): Wind Power and the NIMBY-myth: Institutional Capacity and the Limited Significance of Public Support, Renewable Energy, 21: 49-64. WWEA (2003): Status and Perspectives of Wind Energy. http:/www.wwea.org.
Wynne, B. (1995): Public Understanding of Science, en: Jasanoff, S. et al. (1995): Handbook of Science and Technology Studies, Thousand Oaks: Sage. 